The AsTrophysical JouRnal, 332:L45-L48, 1988 September 15

(C) 1988. The American Astronomical Society. All rights reserved. Printed in U.S.A.

\title{
A NEWLY DISCOVERED IRAS QSO CLOSE TO THE GALACTIC PLANE ${ }^{1}$
}

\author{
Michael A. Strauss \\ Physics and Astronomy Departments, University of California, Berkeley \\ S. D. Kirhakos \\ Cerro Tololo Inter-American Observatory and Instituto de Pesquisas Espaciais \\ AND \\ AmOS YAHIL \\ Astronomy Program, State University of New York at Stony Brook \\ Received 1988 April 22; accepted 1988 June 16
}

\begin{abstract}
As part of a complete survey of IRAS galaxies close to the Galactic plane, we have discovered a bright QSO, at a redshift $z=0.0571$ and a Galactic latitude of $-9^{\circ} 2$. Its observed $V$ magnitude is 13.55 , making it the fourth brightest QSO in the sky, and the Galactic reddening is estimated to be $E(B-V) \approx 0.23$, giving the QSO an absolute magnitude of $M_{V}=-24.87\left(4 \pi r^{2} v F_{v} \approx 5 \times 10^{11} L_{\odot} ; H_{0}=50 \mathrm{~km} \mathrm{~s}^{-1} \mathrm{Mpc}^{-1}\right)$. Direct imaging and spectroscopy show that the object is surrounded by low surface-brightness fuzz, with strong lines of [O III]. The fuzz is some 2.4 mag fainter than the QSO in $R$. Unlike other QSOs recently discovered by $I R A S$, this object does not have a strong infrared excess, and has normal optical colors for a nearby QSO.

Subject headings: infrared: sources - quasars
\end{abstract}

\section{INTRODUCTION}

With the advent of the data base from the Infrared Astronomical Satellite (IRAS), the possibility of selecting QSOs based on their infrared flux has become a reality. Some 74 previously known QSOs were detected by IRAS in at least one of the four bands, although only 21 are bright enough to be seen in the Point Source Catalog, according to Neugebauer et al. (1986). Several new QSOs have been discovered in the data base serendipitously (Beichman et al. 1986; Vader and Simon 1987; Vader et al. 1987), while surveys of warm IRAS sources specifically designed to find active objects have been quite successful (Carter 1984; Osterbrock and de Robertis 1985; De Grijp et al. 1985, 1987; Kailey and Lebofsky 1988; Low et al. 1988). In this Letter, we describe spectroscopic and direct imaging observations of a new bright IRAS-selected QSO, at $\alpha=9^{\mathrm{h}} 14^{\mathrm{m}} 59^{\mathrm{s}} 3$, $\delta=-62^{\circ} 06^{\prime} 55^{\prime \prime}$ (IRAS Point Source Catalog coordinates, epoch 1950), or Galactic coordinates $l_{I I}=280^{\circ} 6, b_{I I}=-9^{\circ} .2$. Its low redshift $(z=0.0571)$ and bright optical magnitude $(V=13.55)$ make it an ideal candidate for further study; it is the fourth brightest QSO in the sky (Hewitt and Burbidge 1987). No data exist for it, to our knowledge, outside of the optical and IRAS bands; presumably it has evaded detection up until now because of its low Galactic latitude. It is near enough that its host galaxy is clearly visible on the ESO Sky Survey plates, and thus reflects the continuity in properties between Seyfert 1 galaxies and QSOs.

\section{DISCOVERY AND SPECTROSCOPY}

As part of a flux-limited redshift survey of IRAS galaxies covering the entire sky (Strauss and Davis 1988a, b; Yahil 1988), we have obtained optical spectra of nonstellar objects selected from the IRAS Point Source Catalog (1985, hereafter PSC) close to the Galactic plane. The object I09149-6206 was

${ }^{1}$ Based in part on observations done at Cerro Tololo Inter-American Observatory, operated by the Association of Universities for the Advancement of Astronomy. observed spectroscopically in a 200 s exposure on 1987 December 5 UT using the $1.5 \mathrm{~m}$ telescope at Cerro Tololo InterAmerican Observatory. The detector used was a GEC CCD mounted at the Cassegrain focus, using a reflection grating of 300 lines per $\mathrm{mm}$, with a resolution of $\sim 8 \AA$ and a spectral coverage of $5640-8060 \AA$. The broad $\mathrm{H} \alpha$ line showed it to be an interesting object, and it was reobserved in an $800 \mathrm{~s}$ exposure the next night with the grating tilted further to the blue, covering 4840-7290 $\AA$. Figure $1 a$ shows a co-addition of the two exposures. The spectra were wavelength-calibrated with observations of a He-Ar lamp, and flux-calibrated with observations of the standard stars LTT 377 and LTT 3864 (Baldwin and Stone 1984). The observations were made through a $3^{\prime \prime}$ slit, and both nights were photometric. However, the slit was aligned $\sim 45^{\circ}$ away from the parallactic angle (Filippenko 1982) during the blue observation, and thus there may be some differential slit loss in the blue.

The spectrum of the object is typical of Seyfert 1 galaxies and QSOs, with broad Balmer lines (note that [N II] is completely blended with $\mathrm{H} \alpha$ ), broad $\mathrm{He}$ I $\lambda 5876$, and strong narrow [O III] $\lambda \lambda 4959,5007$. The $\lambda 4570$ and $\lambda \lambda 5190,5320$ blends of permitted $\mathrm{Fe}$ II emission are also strong. Table 1 gives the parameters of Gaussian fits to lines in the blue spectrum; the equivalent width of the $\mathrm{Fe}$ II emission was found simply by integrating under the curve, rather than fitting a functional form. The heliocentric redshift measured from the peak of the [O III] lines is $z=0.0571 \pm 0.0001$, or $v=17,120 \pm 35 \mathrm{~km} \mathrm{~s}^{-1}$. The slit-guiding error dominates the uncertainty in the redshift.

The blue spectrogram of the object shows it to be slightly extended along the slit. To extract the spectrum of this fuzz, the following was done. We fitted Gaussian profiles to the central five pixels of each row of the QSO spectrogram and the spectrogram of the standard star LTT 3864. The sky had already been subtracted from these frames. We then summed the total counts in each row in 30 pixels $\left(57^{\prime \prime}\right)$ centered on the QSO and the star. We scaled the latter by the ratio of the peaks of the fitted Gaussians, and subtracted it from the total counts in 

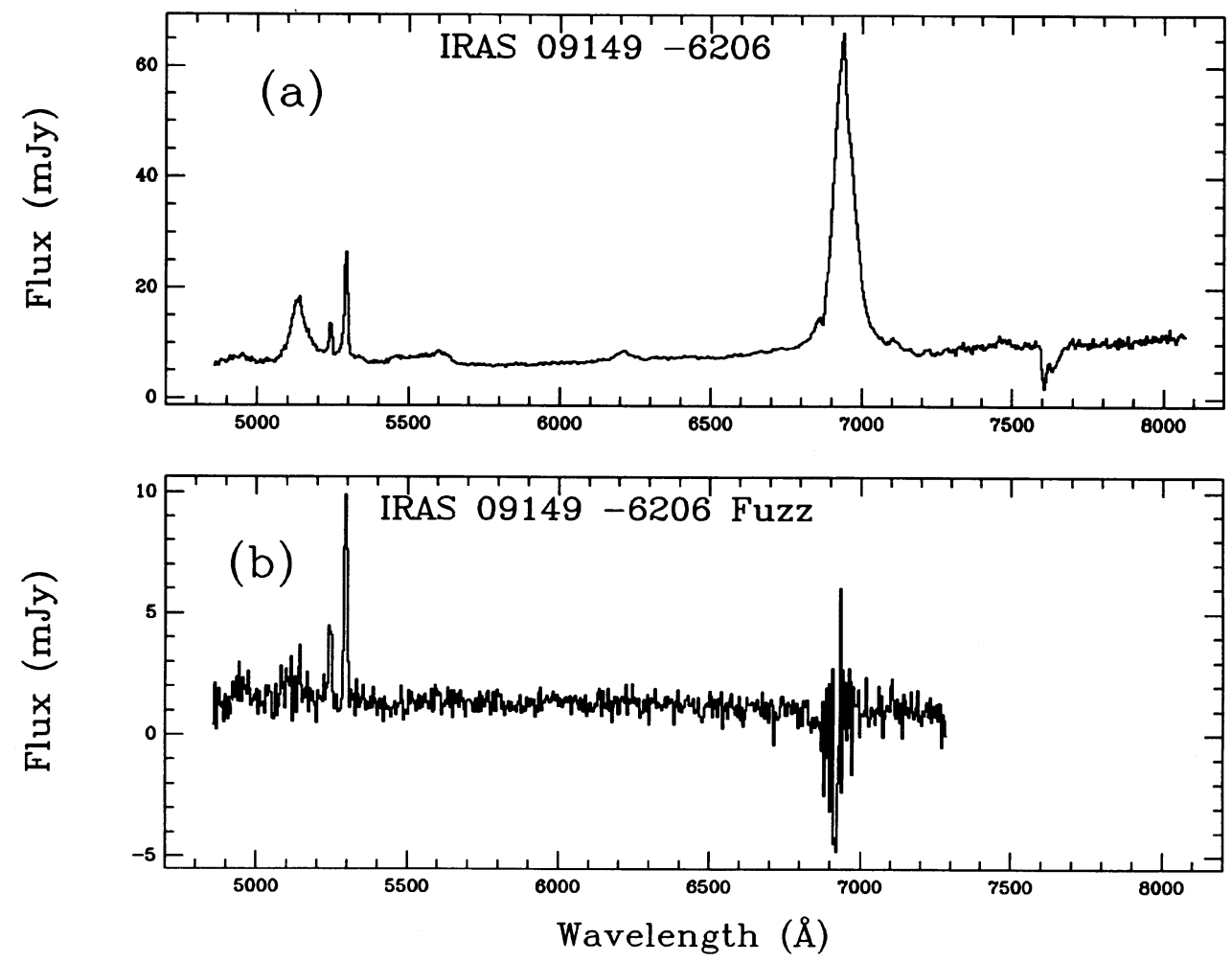

FIG. 1. - (a) A co-addition of the red and blue spectra of the QSO. The absorption features at 6850 and $7600 \AA$ are due to telluric $\mathrm{O}_{2}$ absorption. (b) The spectrum of the fuzz surrounding the QSO. The method of extraction of the spectrum is described in the text.

the row of the QSO. This difference is then an approximation to the counts contributed by the fuzz, assuming that the QSO nucleus is absolutely stellar and that the seeing had not changed between the observations of the object and star.

The resulting spectrum of the fuzz is shown in Figure $1 b$. The continuum is well detected, as are the [O III] lines, while the Balmer lines have completely vanished. The negative feature at $\mathrm{H} \alpha$ is an indication that our fuzz extraction technique is not ideal. Measured parameters for the [O III] lines are given in Table 1.

\section{DIRECT IMAGING}

We obtained direct images in the Johnson $U, B$, and $V$ bands, and in the Kron-Cousins $R$ and $I$ bands on 1987 December 21, using the RCA No. 4 chip, and on 1988 January 6 , using the Texas Instruments No. 1 chip, on the $0.91 \mathrm{~m}$ telescope at CTIO. The exposure times ranged from $40 \mathrm{~s}$ to 300 s. Both chips have pixels that subtend 0"496 square on the sky. The seeing was 1".4 on both nights, and both nights were photometric. The frames were bias-subtracted, and flat-fielded

TABLE 1

Parameters of Fits to Line in Blue Spectrum

\begin{tabular}{|c|c|c|c|c|}
\hline Line & $\begin{array}{l}\text { Observed Wavelength } \\
(\AA)\end{array}$ & $\begin{array}{l}\text { Velocity } \\
\left(\mathrm{km} \mathrm{s}^{-1}\right)\end{array}$ & $\begin{array}{l}\text { Velocity Widtha } \\
\left(\mathrm{km} \mathrm{s}^{-1}\right)\end{array}$ & $\underset{(\AA)}{\text { Equivalent Width' }}$ \\
\hline \multicolumn{5}{|c|}{ Measured Properties of the Emission Lines of the QSO } \\
\hline 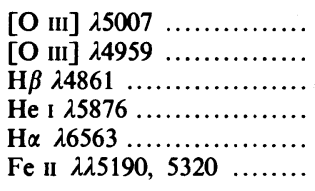 & $\begin{array}{l}5292.8 \pm 0.2^{c} \\
5242.0 \pm 0.6 \\
5136.7 \pm 0.8 \\
6209.8 \pm 1.9 \\
6935.7 \pm 0.4 \\
\cdots\end{array}$ & $\begin{array}{c}17125 \pm 12 \\
17116 \pm 34 \\
16982 \pm 48 \\
17053 \pm 100 \\
17032 \pm 20 \\
\ldots\end{array}$ & $\begin{array}{c}570 \pm 30 \\
600 \pm 100 \\
3500 \pm 100 \\
3000 \pm 200 \\
3350 \pm 50 \\
\cdots\end{array}$ & $\begin{array}{r}36 \pm 2 \\
13 \pm 2 \\
89 \pm 4 \\
13 \pm 1 \\
452 \pm 8 \\
40\end{array}$ \\
\hline \multicolumn{5}{|c|}{ Measured Properties of the Emission Lines of the Fuzz } \\
\hline $\begin{array}{l}{[\mathrm{O} \text { III] } \lambda 5007 \ldots \ldots \ldots \ldots \ldots} \\
{[\mathrm{O} \text { III }] \lambda 4959 \ldots \ldots \ldots \ldots \ldots}\end{array}$ & $\begin{array}{l}5294.2 \pm 0.3 \\
5243.5 \pm 0.8\end{array}$ & $\begin{array}{l}17206 \pm 17 \\
17208 \pm 47\end{array}$ & $\begin{array}{l}430 \pm 50 \\
470 \pm 150\end{array}$ & $\begin{array}{l}71 \pm 6 \\
28 \pm 6\end{array}$ \\
\hline
\end{tabular}


TABLE 2

PHOTOMETRY OF QSO

\begin{tabular}{|c|c|c|c|}
\hline Band & $\begin{array}{l}\text { Observed } \\
\text { Magnitude }\end{array}$ & $\underset{(\mathrm{mJy})}{F_{\mathrm{v}}^{\mathrm{a}}}$ & $\begin{array}{c}\nu F_{v} \\
\left(10^{-11} \mathrm{ergs} \mathrm{cm}^{-2} \mathrm{~s}^{-1}\right)^{\mathrm{a}}\end{array}$ \\
\hline \multicolumn{4}{|c|}{ QSO Broad-Band Fluxes Within a 6' Aperture } \\
\hline$\ldots$. & $13.44 \pm 0.2$ & 20.6 & 18.5 \\
\hline$B$................ & $14.07 \pm 0.06$ & 23.41 & 16.0 \\
\hline$V \ldots \ldots \ldots \ldots$ & $13.55 \pm 0.01$ & 26.86 & 14.7 \\
\hline$R \ldots \ldots \ldots \ldots \ldots$ & $13.01 \pm 0.02$ & 33.46 & 15.7 \\
\hline$I \ldots \ldots \ldots \ldots \ldots$ & $12.74 \pm 0.02$ & 30.2 & 11.5 \\
\hline $12 \mu \mathrm{m} \ldots \ldots \ldots . .$. & $\ldots$ & 490 & 12.3 \\
\hline $25 \mu \mathrm{m} \ldots \ldots \ldots . .$. & $\ldots$ & 1170 & 14.0 \\
\hline $60 \mu \mathrm{m} \ldots \ldots \ldots$ & $\ldots$ & 2400 & 12.0 \\
\hline $100 \mu \mathrm{m} \ldots \ldots \ldots$ & $\ldots$ & 1820 & 5.46 \\
\hline
\end{tabular}

Fuzz Broad-Band Fluxes Within a 12' Aperture

\begin{tabular}{lccc}
\hline$U \ldots \ldots \ldots \ldots \ldots$ & $\ldots$ & $\ldots$ & $\ldots$ \\
$B \ldots \ldots \ldots \ldots \ldots$ & 17.0 & 1.6 & 1.1 \\
$V \ldots \ldots \ldots \ldots \ldots \ldots$ & 15.7 & 3.7 & 2.0 \\
$R \ldots \ldots \ldots \ldots \ldots$ & 15.4 & 3.8 & 1.8 \\
$I \ldots \ldots \ldots \ldots \ldots$ & 15.0 & 3.6 & 1.4 \\
\hline
\end{tabular}

a Corrected for galactic reddening.

with observations taken of a quartz lamp reflected off the inside of the dome. The photometric calibration was done with observations of stars from Graham (1982) and Walker (unpublished; an update to Menzies, Banfield, and Laing 1980). The $I$ band image from the December run is shown in Figure 2 (Plate L4); the fuzz around the object is clearly visible here. The results of the photometry through a circular 6" aperture are given in the second column of Table 2 , along with the fluxes reported in the four IRAS bands in the PSC. No $K$ corrections or IRAS color corrections (IRAS Explanatory Supplement 1985) have been applied to any of these values. The IRAS fluxes listed in the PSC are listed as accurate to between $4 \%$ and $8 \%$ for the 12 and $25 \mu \mathrm{m}$ bands, and between $8 \%$ and $12 \%$ for the 60 and $100 \mu \mathrm{m}$ bands. The PSC correlation coefficients indicate that the source is unresolved. Thus although the cirrus flags indicate the presence of infrared cirrus in the region, these fluxes are probably not contaminated by cirrus cmission.

The maps of Burstein and Heiles (1982) allow us to obtain a rough estimate of the extinction by looking at $b=-10^{\circ}$ at the same Galactic longitude as the QSO. They give $E(B-V)=0.21$ at this position. Alternatively, Kerr et al. (1986) give an integrated $\mathrm{H}$ I column density at the coordinates of the QSO of $1.4 \times 10^{21} \mathrm{~cm}^{-2}$, which gives $E(B-V)=0.23$ for a normal gas-to-dust ratio. We adopt the latter value here. In the third column of Table 2 we correct the optical fluxes for this reddening, converting the magnitudes to fluxes using the absolute calibration of Bessell (1979). In Figure 3 we plot the quantity $4 \pi r^{2} v F_{v}$ from optical to infrared. We have used a Hubble constant of $50 \mathrm{~km} \mathrm{~s}^{-1} \mathrm{Mpc}^{-1}$ and $q_{0}=0$. This source is not in a field observed by the Einstein X-ray satellite, nor has it been included in the Parkes or Molonglo surveys of radio sources in the Southern Hemisphere. The spectral energy distribution is very flat; this is not a QSO with a strong infrared excess, although it does have the warm infrared colors of the active galaxies of Kailey and Lebofsky (1988) and Low et al. (1988). Sanders et al. $(1988 a, b)$ discuss the spectral energy distribution of a number of active galaxies, QSOs and ultraluminous infrared galaxies, and show that they form a continuum in ratio of infrared to optical luminosity. The more infrared-bright objects are very dusty, and presumably are QSOs in the process of formation. In the scenario of Sanders $e t$ al., our object is a full-fledged QSO that has blown away all of the dust from its birth; the optical colors are quite normal for optically selected QSOs (Sandage 1973). However, this object has a $60-100 \mu \mathrm{m}$ spectral slope of 0.54 , which is larger than that of any other QSO measured by IRAS (Neugebauer et al. 1986); this may signify significant contamination at $60 \mu \mathrm{m}$ from the host galaxy.

Figure 2 clearly shows that the QSO is surrounded by a host galaxy. A point-spread function was found for each frame from several unsaturated stars in the field, using the program DAOPHOT (Stetson 1987), and all stellar images were sub-

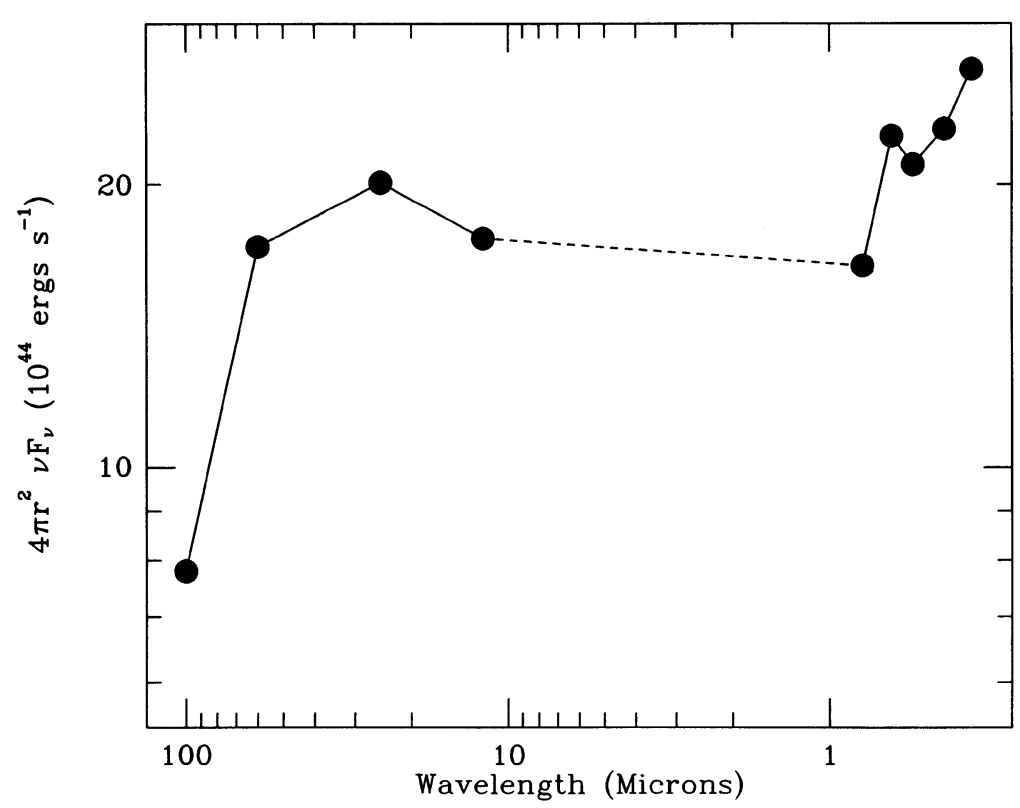

FIG. 3. - The spectral energy distribution of the QSO from broad-band optical and infrared data. The data have been corrected for Galactic reddening. 
PLATE L4 
tracted from the frame, presumably leaving only the light of the underlying galaxy. The results of this procedure are shown in Table 2, in which we present photometry of the fuzz within a 12 " aperture centered on the QSO. The galaxy is 2.4 mag fainter than the QSO in $R$, roughly consistent with the continuum ratio of Figures $1 a$ and $1 b(1.8 \mathrm{mag})$. It is impossible to quantify the errors on the quantities in the second half of Table 2 , given the crudeness of the subtraction technique, but the numbers are probably good to no better than half a magnitude.

\section{DISCUSSION}

The spectrum in Figure $1 b$ is quite similar to spectra of fuzz around other high-luminosity QSOs (Boroson, Persson, and Oke 1985 and references therein); the strong [O III] lines imply that the underlying galaxy is gas-rich, with its gas photoionized by the power-law spectrum of the central QSO. This object does not, however, fit neatly into the categories of Boroson, Persson, and Oke (1985), who find that the majority of luminous QSOs with fuzz dominated by [O III] emission have weak Fe II emission, and very broad $\mathrm{H} \beta$.

The absolute magnitude of the fuzz is $M_{V} \approx-22$ $\left(4 \pi r^{2} v F_{v} \approx 7 \times 10^{10} L_{\odot}\right)$ after correction for reddening, somewhat brighter than $L_{*}$ (Davis and Huchra 1982), and comparable to host galaxies of other nearby QSOs (Hutchings 1983 and references therein). The fuzz is detected above sky to $\sim 10^{\prime \prime}$ from the QSO, or $16 \mathrm{kpc}$ at that redshift, but deeper photometry is needed to compare the size of the galaxy to the host galaxies of the QSOs of Wyckoff (1981), who find an average metric radius of $\sim 50 \mathrm{kpc}$ at the $26 R \mathrm{mag} \operatorname{arcsec}^{-2}$ level.

Weedman's (1986) cumulative luminosity function of nearby QSOs implies one QSO brighter than $M_{B}=-23.9$ with a redshift less than 0.1 ; our object has $M_{B}=-24.5$ after correction for galactic reddening, making it the most luminous QSO in this redshift range. Its existence is thus roughly consistent with the predictions of the luminosity function.

The brightness and nearness of this QSO make it a prime candidate for further observations. For instance, blue spectra of the fuzz having high signal-to-noise ratios will be relatively easy to obtain and may reveal absorption lines indicative of a young stellar population (Boroson and Oke 1982), and imaging in good seeing conditions will yield important morphological information about the underlying galaxy. Furthermore, the source may be a strong source at X-ray and radio wavelengths (although the strong Fe II emission argues against the latter possibility) and may show variability properties. We call for further observations in all passbands available to further elucidate the nature of this QSO.

Many thanks to Alex Filippenko and Marc Davis for helpful comments and suggestions. The help of Giampaolo Piotto, Kate Ebneter, and Pat McCarthy with the image processing was invaluable. Thanks also to Jorge Bravo and Hernán Tirado for their expert assistance at the telescopes. M. A. S. acknowledges the support of a National Science Foundation Fellowship and a Berkeley Graduate Fellowship.

\section{REFERENCES}

Baldwin, J. A., and Stone, R. P. S. 1984, M.N.R.A.S., 206, 241

Beichman, C. A., Soifer, B. T., Helou, G., Chester, T. J., Neugebauer, G., Gillett, F. C., and Low, F. J. 1986, Ap. J. (Letters), 308, Li.

Bessell, M. S. 1979, Pub. A.S.P. $91,589$.

Boroson, T. A., and Oke, J. B. 1982, Nature, 296, 397.

Boroson, T. A. Persson, S. E. and Oke, J. B. 1985, Ap. J., 293, 120

Burstein, D. and Heiles, C. 1982, A.J., 87, 1165 .

Carter, D. 1984, Astr. Express, 1, 61.

Carter, D. 1984, Astr. Express, 1, 61.

Davis, M., and Huchra, J. P. 1982, Ap. J., 254, 437.

De Grijp, M. H. K., Miley, G. K., and Lub, J. 1987, Astr. Ap. Suppl., 70, 95

De Grijp, M. H. K., Miley, G. K., Lub, J., and de Jong, T. 1985, Nature, 314, 240.

Filippenko, A. V. 1982, Pub. A.S.P., 94, 715

Graham, J. A. 1982, Pub. A.S.P., 94, 244.

Hewitt, A., and Burbidge, G. 1987, Ap. J. Suppl., 63,1.

Hutchings, J. B. 1983 , Pub. A.S.P., $95,799$.

IRAS Catalogs and Atlases, Explanatory Supplement. 1985, ed. C. A. Beichman, G. Neugebauer, H. J. Habing, P. E. Clegg, and T. J. Chester (Washington D.C.: U.S. Government Printing Office).

IRAS Point Source Catalog. 1985, Joint IRAS Science Working Group (Washington, D.C.: U.S. Government Printing Office) (PSC).

Kailey, W. F., and Lebofsky, M. J. 1988, Ap. J., 326, 653.

Kerr, F. J., Bowers, D. F., Jackson, P. D., and Kerr, M. 1986, Astr. Ap. Suppl., 66, 373 .

Low, F. J., Huchra, J., Kleinmann, S. G., and Cutri, R. M. 1988, Ap. J. (Letters),

Menzies, J. W., Banfield, R. M., and Laing, J. D. 1980, South African Astr. Obs. Circ., 1, 149.

Neugebauer G., Miley, G. K., Soifer, B. T., and Clegg, P. E. 1986, Ap. J., 308, 815.

Osterbrock, D. E., and de Robertis, M. M. 1985, Pub. A.S.P., 97, 1129.

Sandage, A. 1973, Ap. J., 180, 687.

Sanders, D. B., Soifer, B. T., Elias, J. H., Madore, B. F., Matthews, K., Neugebauer, G., and Scoville, N. Z. 1988a, Ap. J., 325, 74

Sanders, D. B., Soifer, B. T. Elias, J. H. Neugebauer, G., and Matthews, K. 1988 b, Ap. J.(Letters), 328, L35

Stetson, P. B. 1987, Pub. A.S.P., 99, 191.

Strauss, M. A., and Davis, M. 1988a, in IAU Symposium 130, Large-Scale Structure in the Universe, ed. J. Audouze and A. Szalay (Dordrecht: Reidel) in press.

. 1988b, in Proc. Pont. Acad. Sciences Study Week 27, Large-Scale Motions in the Universe, ed. V. Rubin and G. C. Coyne, in press.

Vader, J. P., and Simon, M. 1987, Nature, 327, 304.

Vader, J. P., Da Costa, G. S., Frogel, J. A., Heisler, C. A., and Simon, M. 1987, Astr. J., 94, 847.

Weedman, D. W. 1986, Quasar Astronomy (Cambridge: Cambridge University Press).

Wyckoff, S., Wehinger, P. A., and Gehren, T. 1981, Ap. J., 247, 750.

Yahil, A. 1988, in Proc. Pont. Acad. Sci. Study Week 27, Large-Scale Motions in the Universe, ed. V. Rubin and G. C. Coyne, in press. 327, L41.

Sofia D. Kirhakos: Instituto de Pesquisas Espaciais (INPE/MCT), Depto. de Astrofisica, Caixa Postal 515, 12.200 São José dos Campos, São Paulo, Brazil

Michael A. Strauss: Department of Astronomy, University of California, Berkeley, CA 94720

Amos YAHIL: Astronomy Program, State University of New York, ESS Building, Stony Brook, NY 11794-2100 